\title{
Shift Dependent Skew Quadrupole in Advanced Light Source Elliptically Polarizing Undulators, Cause and Corrections
}

\author{
S. Marks, S. Prestemon, D. Robin, R. D. Schlueter, C. Steier, A. Wolski, J. Y. Jung, O. Chubar
}

\begin{abstract}
Three elliptically polarizing undulators (EPU) are installed and operational at the Advanced Light Source (ALS); the most recent was installed in April '05. Operational experience has shown a variation in electron beam size which correlates with the EPU's magnetic quadrant shifts used to vary polarization. Storage ring electron dynamics studies pointed to the existence of a shift dependent skew quadrupole (SQ) component generated within the EPUs. Detailed magnetic and mechanical measurements demonstrated that the field errors were the result of systematic individual magnetic block displacements which vary with quadrant shift. This paper will discuss the results of electron dynamics studies, magnetic and mechanical measurements, design modifications planned for future EPUs to eliminate the SQ source, and the design and implementation of SQ compensation coils.
\end{abstract}

Index Terms-Elliptically polarizing undulator, skew quadrupole, permanent magnets.

\section{INTRODUCTION}

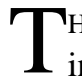
HREE elliptically polarized undulators (EPUs) have been installed at the Advanced Light Source (ALS) of the Lawrence Berkeley National Laboratory [1]. All have a 50 $\mathrm{mm}$ period and are based upon the Apple-II design.[2] The EPUs feature a magnetic structure with four permanent magnet based quadrants as shown schematically in Fig. 1. Q2 and Q4 are fixed, and Q1 and Q3 are translated parallel to the magnetic axis in order to vary the relative strength of the horizontal and vertical periodic magnetic fields, and thus control the ellipticity of the electron trajectory and polarization of the resultant radiation. The range of translation is $\pm \lambda / 2$, where $\lambda$ is the period length. Some experiments use

Manuscript received September 20, 2005. This work was supported by the U. S. Deptartment of Energy , under Contract No. DE-AC02-05CH11231.

S. Marks is with the Lawrence Berkeley National Laboratory (LBNL), Berkeley, CA 94720 USA (phone: 510-486-5828, fax: 510-486-4873, email: s_marks@lbl.gov).

S. Prestemon is with LBNL (email: soprestemon@lbl.gov).

R. D. Schlueter is with LBNL (email: rdschlueter@lbl.gov).

C. Steier is with LBNL (email: csteier@lbl.gov).

A. Wolski is with LBNL (email: awolski@lbl.gov).

J. Y. Jungis with LBNL (email: jyjung@lbl.gov).

O. Chubar is with Synchrotron Sololeil, Yvette Cedex, France (email: oleg.chubar@synchrotron-soleil.fr) relatively fast polarization switching. The minimum time to change from left to right circular polarization is about $1.6 \mathrm{~s}$.

The forces on individual magnet blocks vary as a function of quadrant translation, or shift. ${ }^{1}$ This causes a systematic displacement of blocks and a resultant integrated skew quadrupole (SQ) which varies with shift. The undesired result is a variation in beam size due to coupling between horizontal and vertical electron beam displacements caused by the SQ.

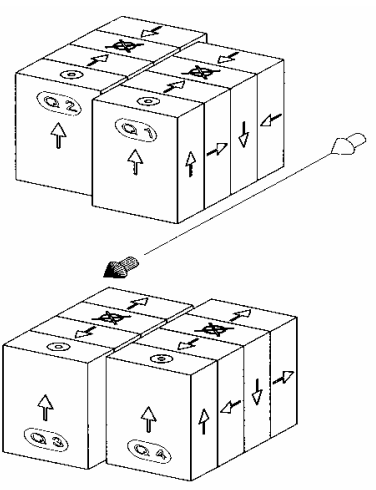

Fig. 1. Schematic of EPU magnetic structure.

Section II of this paper will discuss electron beam and magnetic measurements of the SQ. Section III will discuss the mechanisms generating the SQ and block displacement measurements that confirm the mechanism. Section IV will discuss a trim coil design that has been used to cancel the EPU generated SQ.

\section{SKew Quadrupole MEASUREMENTS}

Variation in the source size in several experiments at various ALS beamlines was the first indication of an SQ. Response matrix analysis [3] determined that a variation in the vertical electron beam size was due to a SQ within the EPUs.

Fig. 2 shows the SQ magnitude as a function of quadrant shift as determined from beam measurements on the first two installed EPUs. Note that the shape of the SQ variation for the two devices are very similar. A different pattern of internal magnetic errors will result in a vertical shift of SQ vs. $x$

\footnotetext{
${ }^{1}$ The term shift will be used to refer to the case where Q1 and Q3 quadrant translations are equal.
} 
without changing its shape; therefore, the vertical offset between the graphs of the two devices is not significant. Detailed SQ magnetic measurements were done for the third device and are shown in Fig. 3. The shape of the SQ vs $x$ measured for EPU3 is similar to those inferred from beam measurements for EPU1 and EPU2.The magnitude, however, is somewhat different. This may be due to slight mechanical differences or different measurement techniques.

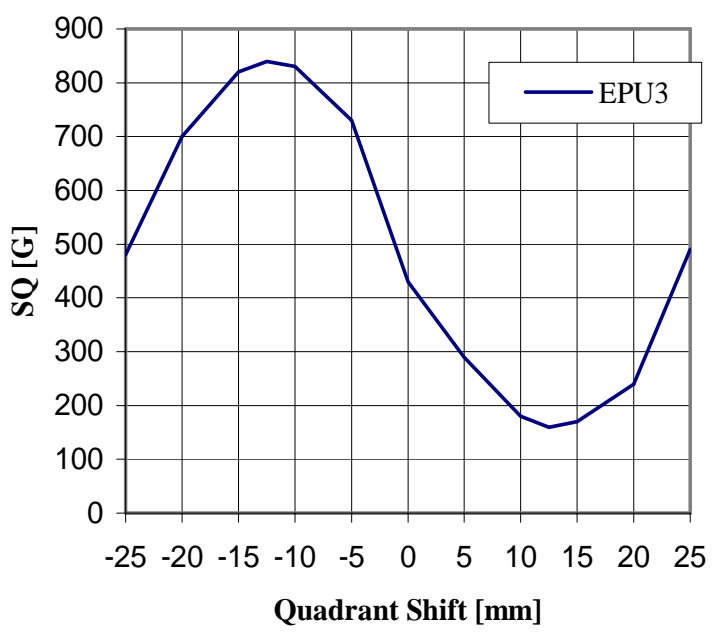

Fig. 2. Magnetic measurement of skew quadrupole variation with quadrant shift, $d s$

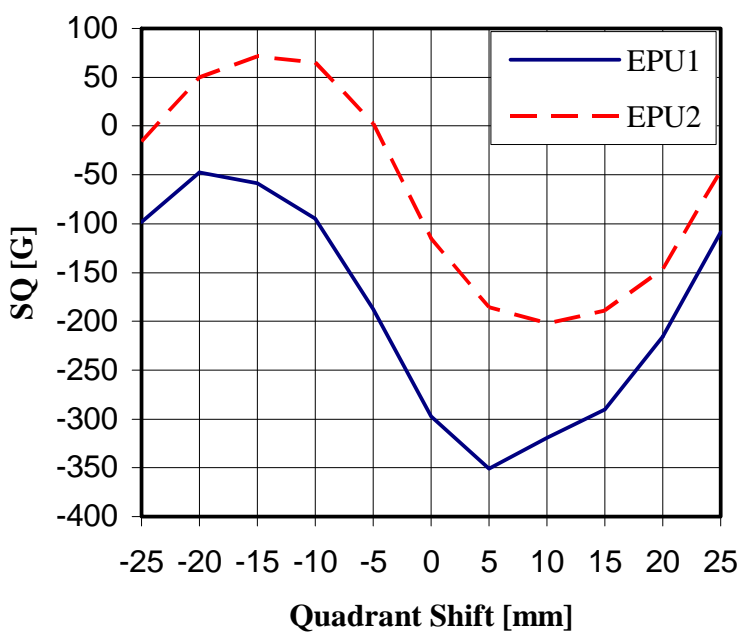

Fig. 3. Response matrix determination of SQ variation with quadrant shift

\section{Skew Quadrupole Mechanism}

The fact that the SQ functional dependence on shift is very similar for all three of the EPUs, suggests that the effect is systematic and inherent to our basic design. The existence of a SQ per se is not surprising. It can be the result of accumulated magnetic errors or magnetic shimming. The challenge was to find a mechanism that results in a SQ that varies with shift. The following possible mechanisms were considered:

- Variation in neighboring permeability, $\mu$

- Proximity to high permeability steel.
- Demagnetization and hysteresis.

- Motion of the magnetic structure.

\section{A. Variation in permeability}

The relative permeability of Nd-Fe-B magnetic blocks is typically 1.05 and 1.15 , parallel and perpendicular to the easy axis, respectively. Since each of the magnetic quadrants is composed of a sequence of magnets that are alternately oriented parallel and perpendicular to the translation axis, the magnetic neighborhood of an individual block varies with shift. Additionally, as quadrants translate, half of the end blocks move from positions near magnet blocks in adjacent quadrants to where they are surrounded by air, with $\mu=1$. This could result in integrated field variations. In fact, it has been clearly demonstrated that a shift dependent variation in integrated dipole occurs in EPUs due to the variation in the magnetic environment of end blocks [4]. However, in the periodic section of the magnetic structure, the integral effect of individual blocks' variation in exposure to $\mu=1.05$ and 1.15 material cancels. The integrated dipole effect of end blocks is reproduced in magnetic models using the Radia [5] code. Magnetic models show no effect on integrated gradients and higher order terms with shift.

\section{B. Proximity to high permeability steel}

The EPU support and drive system incorporates high permeability steel, and slightly magnetic stainless steel bolts are incorporated within the magnetic structure. These could have an effect if the proximity of magnetic blocks to steel varies with shift, and if they are close enough to make a difference. The distance between the magnetic structure and steel support posts (the closest steel component of the support structure) is large compared to the magnetic period length. If the steel supports influence the EPUs magnetic field, then measurable magnetic fields would exist in the vicinity of the structure. Furthermore, the magnitude would have to vary with quadrant shift to account for the observed SQ. The measured fields near the supports are indistinguishable from background environmental fields, and show no variation with shift. Radia models also show no effect.

Fig. 4 shows the structure of a magnetic module. The tie rods that hold the group of four magnetic blocks together, as well as the horizontal and vertical bolts which tie the module to the backing beam are made of series 300 stainless steel, which were determined to be slightly magnetic (i.e. $\mu>1$ ). However, even though they may have some influence on the magnetic field, they are not a likely candidate for shift dependent variation, since they are uniformly distributed throughout the magnetic structure. Radia analysis, which exaggerated the influence of the fasteners by modeling them as high permeability low carbon steel, showed no significant magnetic effect, and no shift dependence.

\section{Demagnetization and hysteresis}

The magnetic fields within magnetic blocks vary with quadrant shift. Demagnetization will occur if reverse fields of 
sufficient magnitude are induced. However, if demagnetization were responsible for the repeatable shift dependent SQ, a hysteresis loop would have to consist of remagnetization as well as demagnetization. This requires very large magnetic field excursions. Radia models showed that no such hysteresis loops occur. However, the models were used to identify magnetic block regions with the largest magnetic excursions and closest to demagnetization. A Helmholtz coil was used to measure block magnetization before and after exposure to dipole fields characteristic of the maximum excursion. The measurements showed no demagnetization and no hysteresis.

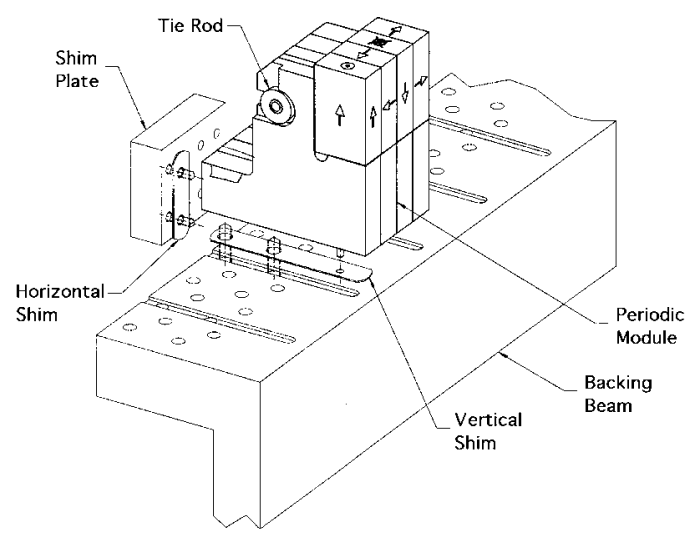

Fig. 4. Magnetic module.

\section{Motion of magnetic structure}

Fig. 4 shows vertical and horizontal shims that are installed for each magnetic block. The shim thickness is used to adjust static horizontal and vertical block displacements for the purpose of magnetic field tuning. Fig. 5, for example, shows the perturbation to $I_{x}(x)=\int_{-\infty}^{\infty} B_{x}(x, z) d z$ due to a horizontal block displacement. Note that a horizontal block displacement results in a perturbation to $I_{x}$ as well as $d I_{x} / d x$, which corresponds to a SQ. If blocks are displaced horizontally as a function of quadrant shift, this could be the cause of the shift dependent SQ.

The horizontal force acting on full quadrants has a large variation with shift. The force between two adjacent quadrants is repulsive when they are aligned (shift $=0$ ); the force is attractive at full shift (shift $=\lambda / 2$ ). This results in relative horizontal motion between adjacent quadrants on the order of $0.5 \mathrm{~mm}$. However, due to the periodic magnetic structure, the contribution from successive blocks to both $I_{x}$ and SQ cancels and the effect on integrated magnetic fields is zero.

Let us now consider the effect of motion of individual blocks. A shift dependent SQ of the correct magnitude would be caused by the following shift correlated block motions:

1. A single block motion of $\pm 1.5 \mathrm{~mm}$.

2. A random distribution of displacements with $\sigma \sim 45$ $\mu \mathrm{m}$.

3. Motion of vertical blocks of up ( $\uparrow$ ) and down ( $\downarrow$ ) orientations are anti-correlated; with every $\uparrow$ moving $\pm 3 \mu \mathrm{m}$ and every $\downarrow$ moving $\mp 3 \mu \mathrm{m}$.

The required displacement magnitude for scenario 1 is too large to not be noticed. The required magnitude for scenario 2 is also large, and the fact that three devices behave the same makes random displacement unlikely. Direct measurements of individual magnet block displacements demonstrated that scenario 3 is indeed responsible for the observed SQ.

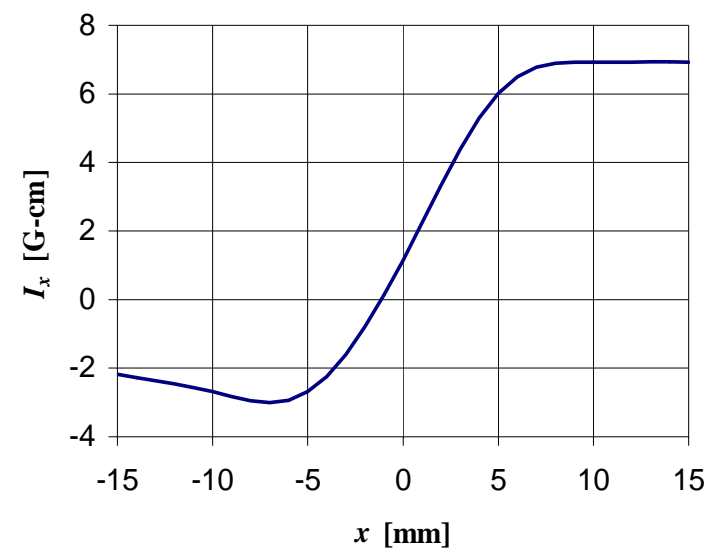

Fig. 5. Perturbation of $I_{x}$ due to a unit $(0.025 \mathrm{~mm}$ shim increment) horizontal displacement of a vertically oriented block in quadrant 1 .

Fig. 6 shows measurements of differential horizontal motion of $\uparrow$ and $\downarrow$ oriented blocks within a single magnetic module, typical for each of the four quadrants. The magnitudes are of the right order to account for the observed SQ, and the signs for the four quadrants are such that they are additive. A Heidenhain-Certo CT 60 length gauge was used for the measurements. The gauge was rigidly attached via a bracket to the backing beams at various locations to measure horizontal displacement of individual blocks as a function of quadrant shift. The measurements were repeatable to $\pm 0.5 \mu \mathrm{m}$.

The differential motion of $\uparrow$ and $\downarrow$ oriented blocks, as shown in Fig. 6, corresponds to rotation of the four block magnetic modules, shown in Fig. 4. Axial components of force, which have the correct functional variation with shift, in conjunction with module mounting, produce a torque on modules resulting in rotation.

For future EPU implementations, we plan to mount blocks individually to backing beams, eliminating the four block module grouping, and thus eliminating rotation and differential motion of $\uparrow$ and $\downarrow$ oriented blocks. Additionally we will stiffen the structure to limit horizontal deflection of individual blocks.

\section{SKEW QUADRUPOLE COMPENSATION COIL}

Fig. 7 shows a cross section of the EPU vacuum chamber with trim coil locations marked as numbers 1 through 4 . Note that the coils were retrofitted to existing chambers; their locations are based upon available space between the vacuum chamber and the magnetic structure and are far from the ideal for SQ excitation (coil centers at $\pm 45^{\circ}, \pm 135^{\circ}$ ). The EPU 
magnets fit into the pockets between coils 1 and 2 and coils 3 and 4 . The coil returns are on the outside of the chamber and do not contribute significantly to the on-axis field. Each coil consists of 10 turns. The four coils are connected in series to a 20 A bi-polar power supply.

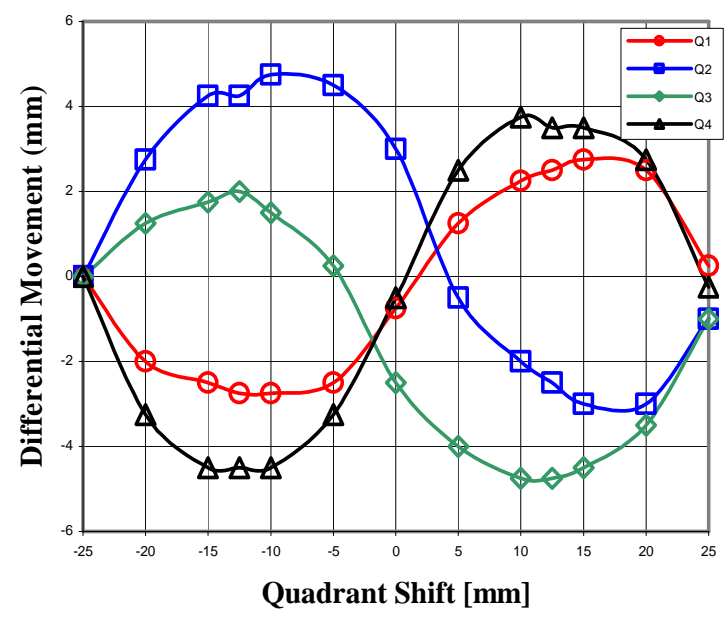

Fig. 6. Differential horizontal motion between $\uparrow$ and $\downarrow$ blocks within a module as a function of quadrant shift.

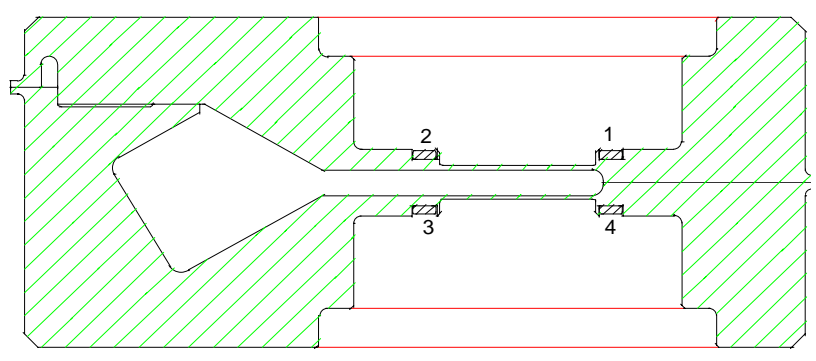

Fig. 7. Cross section of EPU vacuum chamber showing trim coil locations.

The center for coil 1 , designated as a complex number $z=x+i y$, is $z_{c}=3.40+1.44 i$. Centers for coils 2,3 , and 4 are located at $-z_{c}^{*},-z_{c}$, and $z_{c}^{*}$, respectively. For this coil configuration, a SQ excitation consists of all coils with the same polarity. The coils are modeled as coil filaments located at the respective centers. Eq. (1) represents the field harmonics, where $a_{n}$ and $b_{n}$ represent the normal and skew multipoles, respectively. Table I includes the normalized fundamental strength and multipoles calculated for normalization radius $r_{0}=1.0$.

$$
\begin{aligned}
H_{n}^{*} & =i\left(a_{n}+i b_{n}\right)\left(z / r_{0}\right)^{n}=\frac{I}{\pi} \frac{2}{r_{c}^{n+1}} \sin \left((n+1) \theta_{c}\right) z^{n} \\
r_{c}^{2} & =x_{c}^{2}+y_{c}^{2} ; \theta_{c}=\tan ^{-1}\left(y_{c} / x_{c}\right) ; n=1,3,5 \ldots
\end{aligned}
$$

Fig. 8 shows measured results of the trim coils. The compensation for vertical beam size variation is nearly perfect.

TABLE I.

FUNDAMENTAL AND MULTIPOLE STRENGTH.

\begin{tabular}{ccc}
\hline \hline $\boldsymbol{n}$ & $\boldsymbol{b}_{\boldsymbol{n}} / \boldsymbol{I}$ & $\left|\boldsymbol{b}_{\boldsymbol{n}} / \boldsymbol{b}_{1}\right|$ \\
\hline 1 & $3.4 \times 10^{-2}$ & \\
3 & $3.4 \times 10^{-3}$ & $1.0 \times 10^{-1}$ \\
5 & $1.7 \times 10^{-4}$ & $5.0 \times 10^{-3}$ \\
\hline
\end{tabular}

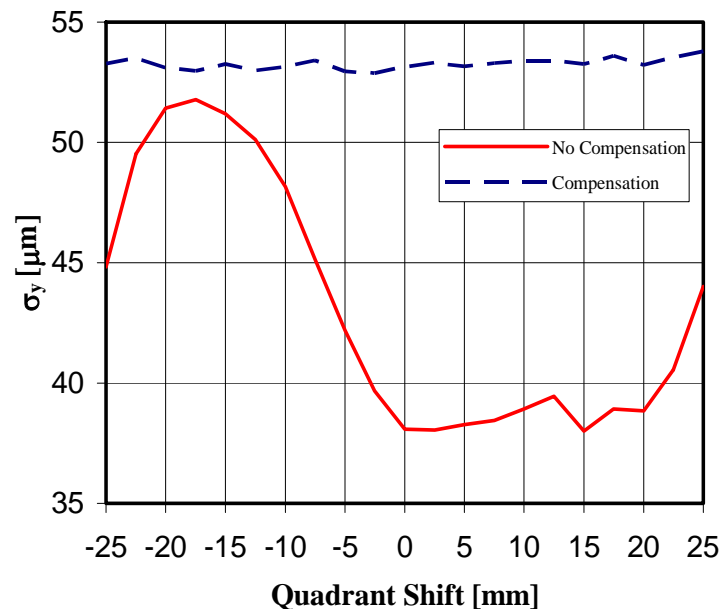

IV. CONCLUSIONS

The EPUs already installed at the ALS have produced valuable scientific results. Future plans include the installation of more EPUs to satisfy the increasing demand for polarization control. The work presented in this paper represents significant progress in the operation of EPUs without negatively impacting electron beam characteristics and stability. The discovery of the mechanical roots of a shift dependent SQ will lead to improved designs to minimize this effect for future devices. The development and implementation of SQ trim coil allows the current devices to operate with minimal impact on the beam.

\section{ACKNOWLEDGMENT}

The authors would like to thank P. Elleaume and J. Chavanne for helpful discussions and suggestions and help with using Radia. We are grateful for W. Wittmer's keen observation of the relative alignment of magnetic modules that was crucial for solving the SQ mystery.

\section{REFERENCES}

[1] S. Marks, et al., "The Advanced Light Source Elliptically Polarizing Undulator”, Proc. 1997 PAC, Vancouver (1998) 3221.

[2] S. Sasaki, "Analysis of a planar variably polarizing undulaotr", Nucl. Instr. And Meth., A 347 (1994) 83.

[3] C. Steier, "Study of Row Phase Dependent Skew Quadrupole Fields in Apple-II Type EPUs at the ALS”, Proc. 2004 EPAC, Lucerne, Switzerland.

[4] J. Chavanne, et al., "End Field Structure For Linear/Helical Insertion Devices”, Proc. 1999 PAC, New York (1999).

[5] O. Chubar, et al., "A 3D Magnetostatics Computer Code for Insertion Devices”, J. Synch. Rad (1998), 5, 481. 